\title{
Análisis tipos de aplicaciones para guarderías, identificando ventajas y desventajas de su uso
}

\author{
Analysis type of application for nurseries, identifying advantages and disadvantages of their use
}

\section{Anayeli Romero Fuentes ${ }^{a}$, Emilio Alejandro Rivera Landero ${ }^{b}$}

\begin{abstract}
:
The objective of this essay is to present an analysis of various applications that a nursery can implement and thus support the Arcoíris nursery located in Apan Hidalgo, in its decision to implement an application of this type or choose to develop its own. tailored to your needs in order to improve trust, communication, and at the same time providing added value to the service with minimal cost).

It seeks to solve the needs that parents have through a technological tool, thanks to the fact that today they have become important and indispensable in our lives, benefiting different factors, whether in education, communication, tourism, medicine, or entertainment, where many companies have managed to grow thanks to the comfort, flexibility and communication that technology offers. The objective of the Arcoíris Nursery is to analyze different technologies already applied in children's rooms and propose the management of an App in order to improve trust, communication, and at the same time providing added value to the service with a minimum cost.
\end{abstract}

\section{Keywords:}

Nurseries, Technology, Applications, Technolpgical tools, Pedagogy.

\section{Resumen:}

Con el presente ensayo se tiene como objetivo presentar un análisis de diversas aplicaciones que una guardería puede implementar y así apoyar a la guardería Arcoíris ubicada en Apan Hidalgo, en su decisión de implementar una aplicación de este tipo o bien optar por el desarrollo de una propia adaptada a sus necesidades con el fin de mejorar la confianza, comunicación, y al mismo tiempo aportando un valor agregado al servicio con un costo mínimo).

De esta forma mediante una herramienta tecnológica se podrá dar solución a las necesidades que tienen los padres de familia, gracias a que hoy en día se tales aplicaciones informáticas han vuelto importantes e indispensables en nuestras vidas, beneficiando distintos factores ya sea en la educación, comunicación, turismo, medicina, o entretenimiento, donde muchas empresas han logrado crecer gracias a la comodidad, flexibilidad y comunicación que ofrece la tecnología.

\footnotetext{
a Universidad Autónoma del Estado de Hidalgo, Escuela Superior Ciudad Sahagún, Estudiante de maestría, Email: anayeli_romero@uaeh.edu.mx

b Autor de Correspondencia, Universidad Autónoma del Estado de Hidalgo, Escuela Superior Ciudad Sahagún, Email: rlandero@uaeh.edu.mx
} 


\section{Palabras Clave:}

Guarderías, Tecnología, Aplicaciones, Herramientas tecnológicas, Pedagogía.

\section{Introducción}

Las guarderías hoy en día son indispensables para mejorar los ingresos del hogar, la educación y desarrollo de los menores, es por ello que en siglo XIX se da origen a guarderías infantiles en Bolonia ciudad de Italia (Cagnolati, 2011).

De acuerdo con (Cagnolati, 2011) la creación de una guardería en Bolonia dio lugar a discusiones y conflictos gubernamentales y religiosos. El nacimiento de las guarderías surgiría de la participación de particulares bajo el control de las autoridades eclesiásticas, a pesar de esto, el conde Carlo Marsili, elaboró un programa para fundar guarderías invitando a todas las clases pudientes de la sociedad para que muestren benevolencia hacia el pueblo plebeyo.

De esta forma y siguiendo al mismo autor, se inauguró la primera guardería el 16 de Junio de 1847 situada en Bolonia Scuole Pie, en la calle Poeti, respondiendo a una necesidad muy apremiante. Sin embargo, la única preocupación de los fundadores era la esfera social y la recaudación de fondos financieros más no el bienestar de los niños en cuanto a temas pedagógicos. (Cagnolati, 2011)

Posteriormente y según (Pacheco Cervantes, 2014) en el siglo XX y gracias a los principios de la democracia liberal que proporcionaron la ideología impulsadora de las mujeres se dio el reconocimiento de la igualdad de derechos entre hombres y mujeres, debido al esfuerzo de muchas mujeres quienes lucharon por su libertad, por sus metas y por su realización personal, lo que proporcionó mayores accesos a servicios como las guarderías.

Actualmente en la mayoría de las familias se presenta la necesidad de que ambos padres (o en las familias monoparentales quien está a cargo de los hijos) tienen la necesidad de salir a trabajar, dejando a sus hijos en estancias infantiles, sin embargo, los padres de familia manifiestan preocupación por dejarlos al cuidado de personas que no conocen como es el caso de la Guardería Arcoíris que se encuentra ubicada en Apan Hgo.

Ante este panorama las guarderías tienen la necesidad, como es el caso de la que nos ocupa en este ensayo, de analizar distintas tecnologías ya aplicadas en estancias infantiles y proponer la gestión de una App con el fin de mejorar la confianza, comunicación, y al mismo tiempo aportando un valor agregado al servicio con un costo mínimo.

De esta forma se busca dar solución a las necesidades que tienen los padres de familia mediante una herramienta tecnológica, gracias a que hoy en día se han vuelto importantes e indispensables en nuestras vidas, beneficiando distintos factores ya sea en la educación, comunicación, turismo, medicina, o entretenimiento, donde muchas empresas han logrado crecer gracias a la comodidad, flexibilidad y comunicación que ofrece la tecnología.

\section{Desarrollo}

Importancia

La importancia de la educación en las edades de 1 a 5 del niño, es de suma importancia pues en ella hay una increíble asimilación de las impresiones que recibe desde el exterior, y es por tanto, la fase en que deben serle suministrados los conocimientos y habilidades mas cercanas a su corazón para así alcanzar su felicidad y su optimo desarrollo en el futuro. (Mahmuod Bani Mustafa, 2017)

El desarrollo infantil temprano (DIT) es la base del capital humano y social y del desarrollo de las naciones. Es por esto que el DIT es un pilar fundamental de los Objetivos de Desarrollo Sostenible. La serie de DIT recién publicada en Lancet indica que es posible implementar programas intersectoriales de DIT basados en evidencia 
a gran escala. La evidencia claramente muestra que estos programas deben ser multifocales, e incluir la salud, nutrición protección social y oportunidades de estimulación y aprendizaje temprano. Según (Pérez Escamilla, 2017) la evidencia también apunta que estos programas deben atender las necesidades de la familia.

Paralelo a esta situación se presenta el fenómeno en donde la mayoría de las familias tienen la necesidad de salir a laborar para mantener una economía estable y aportar una mejor calidad de vida a sus hijos, esto indica que papá y mamá no tienen el tiempo necesario para el cuidado, la educación y desarrollo de los pequeños. Lo cual provoca la demanda de servicios de guarderías particulares y gubernamentales en donde su función se basa en proporcionar atención y cuidado al niño(a) a través de actividades orientadas al cuidado $y$ fortalecimiento de su salud, una sana alimentación y un programa educativo-formativo, acorde a su edad y nivel de desarrollo, para promover su desarrollo integral.

\section{Descripción de control y servicio de Guardería}

La Pedagogía favorece el desarrollo integral de los niños y niñas a través de actividades formativo-asistenciales desde la recepción hasta la entrega; como por ejemplo la higiene, ministración de alimentos y vigilancia del sueño o descanso. Lo escrito por Arnold Gesell, psicólogo y pediatra estadunidense con aportaciones insuperables en el ámbito de la puericultura. (Andrade Soto \& Gonzalez Gonzalez , 2017)

Describe Oscura Blancas, 2015 las conductas de los niños y niñas en diferentes rangos de edad, lo que permite conocer sus características, principalmente en el aspecto psicomotriz que si bien, requiere de elementos cognitivos, se manifiesta en movimientos coordinados del cuerpo.

Existen tres áreas básicas a considerar en el funcionamiento de una guardería, las cuales se describen a continuación:

> Según Oscura Blancas, 2015 la alimentación se enfoca en mantener un esquema alimentario, organizado por regímenes y preparaciones conforme a los requerimientos nutrimentales de acuerdo con la edad, proporcionando menús con platillos y fórmulas lácteas especiales. En caso de encontrar alteraciones en peso o talla, atender las indicaciones del médico por ejemplo la reducción sustantiva de azucares añadidos y grasas saturadas, eliminación de embutidos e incorporar un mayor consumo de frutas $y$ verduras.

> Fomento de la salud Según (Oscura Blancas, 2015) es la encargada de vigilar que el niño(a) al ingreso y durante su estancia en la guardería no presente signos y/o síntomas de enfermedad - lesiones y, de ser el caso, se realizan las acciones necesarias para recibir atención médica; Dar seguimiento a su peso y talla, aplicación de vacunas, informas a los padres sobre los accidentes y/o algún malestar que el niño(a) llegara a presentar y ministra medicamentos que hayan sido prescritos por el médico, con respaldo en receta médica vigente.

$>$ Área de Administración sirve de apoyo para el control de asistencia del personal y de los niños de manera manual.

Tecnología existente

Dentro del análisis del ensayo nos damos cuenta de que no todas las guarderías cuentan con tecnologías que nos faciliten el trabajo guardería-padres de familia, por lo que se realizó una búsqueda en internet, de aplicaciones existentes que se pueden implementar, se encontraron los siguientes aplicaciones y a continuación de describen sus características más importantes:

\section{BabyDay}

- Aplicación interactiva, de control e informativa.

- Presenta acceso a la ficha diaria del niño/a, consultas sobre alimentación, sueño, pañales, avisar al centro infantil 15 minutos antes de recogerlo y chatear con el profesor de tu hijo.

- El control hace fotos a los menores y aplica filtros, dispone de una agenda exclusiva del 
bebé, muestra el calendario de vacunas y avisos, y permite reproducir música para ayudarle a dormir.

- Informa sobre enlaces web para consultas pediátricas especializadas y tiene el acceso a la información del centro infantil, sus actividades y profesores; (BabyDay, 2014)

- 6600 pesos mexicanos - mensuales.

- $\quad$ https://play.google.com/store/apps/details?id=c om.sysia.babysdays

\section{Kínder 360}

- Agiliza el registro de actividades diarias de los niños, proporciona el seguimiento en tiempo real.

- Notificaciones personalizables de los eventos que realiza el alumno en el centro infantil.

- Estadísticas de evolución a partir de datos útiles para la detección temprana de comportamientos irregulares.

- Comunicación bidireccional inteligente que facilita la comunicación entre la escuela y los familiares sin interferir en el trabajo del educador.

- Circulares y menú semanal del centro escolar hacia los padres. (Andrade Soto \& Gonzalez Gonzalez , 2017)

- Costo 520 pesos mexicanos - mensuales.

- $\quad$ https://www.kinder360.com/\#/signin?redirect $=\% 2$ Findex

\section{Kids Care}

- Aplicación móvil diseñada específicamente para garantizar una comunicación fácil, instantánea y sin soporte de papel con los padres de los alumnos.

- Tiene las siguientes características: avisos generales, notificaciones personalizadas, informe de progreso, informe de pagos, eventos, calendarios y evaluación de los padres. (Andrade Soto \& González González, 2017)
- http://www.cienciamx.com/index.php/tecnologia /tic/7983-desarrollan-app-para-monitoreo-deninos-en-estancias-infantiles

\section{Daily Care}

- Seguimiento y monitoreo de las actividades de los menores.

- Agilización del registro de entradas y salidas.

- Reducción de tiempo en el registro de los indicadores.

- Migración de formato digital del cuadernillo de trabajo.

- Reducción en el uso de papel.

- Comunicación de avisos y notificaciones.

- Acceso al historial del menor.

- Graficas fáciles de utilizar y manejar.

- Registro de actividades de los menores, menús, menores inscritos, padres o tutores y personal.

Ventajas de la app en guarderías

La implementación de una aplicación en la administración de una guardería aporta ventajas para quienes deciden utilizarla, entre las cuales encontramos:

- Ideal para persona que se encuentran frecuentemente en un dispositivo móvil.

- Son más prácticas e incluso más rápidas que las webs.

- Aportan comodidad, puesto que miles de aplicaciones hacen que podamos sustituir el tener que acarrear un portátil por una simple tablet o smartphones.

- Aplicaciones que ofrecen la posibilidad de utilizarla sin acceso a Internet.

- Forma habitual para acceder tanto a productos como a servicios.

- Genera mejoras a nivel empresarial. Podemos acceder a documentos y herramientas de nuestra empresa, lo cual mejora el flujo de comunicación y por otro lado lleva implícito un ahorro de costes.

- Pueden ser canales directos que conectan con el usuario para notificar o realizar gestiones como es el caso de las instituciones públicas. 
- No se necesita realizar actualizaciones periódicas.

- Se pueden compartir imágenes, mensajes o documentos privados.

Desventajas de la app en guarderías

- El coste de desarrollo y mantenimiento es mayor.

- No todas las aplicaciones móviles son aceptadas por el mercado.

- Su distribución depende de las tiendas en línea como App Store o Play Store, por lo tanto, tiene que ser descargada e instalada en los smartphones. Por otro lado, también deben de actualizarse para obtener las últimas versiones.

- Las apps consumen espacio, tiempo y en su mayoría datos.

\section{Conclusiones}

En conclusión, nos damos cuenta que las guarderías se han vuelto una prioridad con el fin de mejorar la calidad de vida (padres de familia y pequeños entre 4 meses y 5 años) manteniendo la confianza y satisfacción con los padres de familia, innovando de acuerdo con las necesidades.

La guardería Arcoíris de acuerdo con lo investigado, propone realizar y gestionar una herramienta tecnológica que nos ayuda y beneficie la comunicación entre guardería y padres de familia, notificando la entrada y salida, la alimentación, comportamiento y salud de los pequeños mediante un semáforo. De acuerdo a sus ventajas, desventajas y las características nos damos cuenta de que la aplicación más completa es Baby Day aunque su valor monetario es mayor a comparación a las otras aplicaciones (Kids care, Kinder 360, Daily Care), sin embargo cumple con las expectativas y características que los padres de familia piden como el informe sobre enlaces web para consultas pediátricas especializadas, acceso a la información del centro infantil, sus actividades y profesores, acceso a la ficha diaria del niño/a, consultas sobre alimentación, sueño, pañales, avisar al centro infantil 15 minutos antes de recogerlo y chatear con el profesor de tu hijo. Control de fotos a los menores, agenda exclusiva del bebé, muestra el calendario de vacunas y avisos, y permite reproducir música para ayudarle a dormir entre otras.

La tecnología ha sido de gran impacto en nuestras vidas solucionando, mejorando y optimizando los trabajos a realizar y las herramientas tecnológicas como por ejemplo las apps otorgan un valor agregado a los servicios brindados, mayor seguridad y una atención inmediata.

\section{Referencias}

Andrade Soto, M. A., \& Gonzalez Gonzalez , A. (2017). La importancia de las tecnologías de la Información como Herramientas de Monitoreo para niños en estancias Infantiles: caso Daily Care. Colima: Instituto Tecnologico .

BabyDay. (25 de Marzo de 2014). Ludo y Educa. Obtenido de https://ludoyeducadotcom.wordpress.com/2014/ 03/25/babyday-la-aplicacion-movil-gratuitapara-centros-infantiles/

Cagnolati, A. (2011). El origen de las guarderias infantiles en Bolonia. redalyc.org, 9(13), 91-102.

Mahmuod Bani Mustafa, E. H. (2017). Estudio de la situación de los jardines infantiles publicos en Jordania desde 2000 hasta 2012. Universidad de Granada. Tesis Doctoral. Granada.

Oscura Blancas, L. (Agosto de 2015). Servicio de Guardería para el Desarrollo Integral Infantil. Instituto Mexocano del Seguro Social Coordinación del Servicio de Guardería para el Desarrollo Integral Infantil.

Pacheco Cervantes, M. S. (13 de Marzo de 2014). Proyecto de emprendimiento de una guarderia infantil en centro comercal "Chiquihouse". Univerdad Católicade Santiago de Guayaquil.

Pérez Escamilla, R. (2017). Estancias infantiles y desarrrollo onfantil en México: avances y retos. Boletin Médico del Hospital Infantil de México, 84-85. 